\title{
ADMINISTRACIÓN DEL PROYECTO “DESARROLLO DE CAPACIDADES HUMANAS", MEDIANTE LAS TÉCNICAS ADMINISTRATIVAS CPM Y PERT, URACCAN - SIUNA, 2012
}

Yader Antonio Ordoñez Aguilar ${ }^{[1]}$ Eddy Alberto Zamora Díaz ${ }^{[2]}$ Javier Osmar Artola García[3]

\section{Resumen}

Esta investigación ha aplicado el Método de la Ruta Crítica (CPM) y las Técnicas de Evaluación y Revisión de Programas (PERT) en la administración del proyecto: "Desarrollo de capacidades técnicas y humanas para la implementación del plan de desarrollo regional con identidad en el sector de Las Minas", ejecutado en Siuna, 2012, por la URACCAN. Es un estudio aplicativo retrospectivo con un enfoque cuantitativo enmarcado en las técnicas administrativas de proyecto CPM y PERT mediante el programa WinQSB, en la administración del proyecto en estudio.

De acuerdo a los resultados, todo el proyecto puede concluirse en 51.3 meses, equivalente a 4 años y 3 meses, teniendo 12 actividades críticas, las cuales representan que un retraso en cualquiera de estas ocasionaría un retraso en todo el proyecto. El costo total de las actividades de este proyecto es de 617,863.00 euros y el costo total de la ruta crítica es de 231,585.00 euros. El proyecto incurre en otros costos, tales como: mano de obra, transporte, servicios de consulta, evaluación, entre otros, que también forman parte del presupuesto total del proyecto, siendo este de 1,040,251.00 euros. El proyecto necesitó más tiempo para poder concluir cada una de las actividades, ya que en su planificación inicial estaba programado a terminarse en 3 años, lo cual no se cumplió porque tuvieron que solicitar una prórroga de tres meses para la conclusión total del mismo. La ventaja en la aplicación del Método de la Ruta Crítica, es que dio la perspectiva para tener la información del proyecto de manera organizada y lista para cualquier revisión; una de las principales desventajas es que se tuvo que organizar todas las actividades con respecto a sus predecesores inmediatos y tener conocimiento del método, así como el programa WinQSB para evitar resultados adversos.

Palabras clave: Técnicas administrativas; Programa WINQSB; actividades críticas; ruta crítica; predecesores inmediatos.

\footnotetext{
[1] Licenciado en Administración de empresa. Apoyo Administrativo URACCAN Las Minas. Correo: ordoezyader@yahoo.com

[2] Licenciado en Administración de Empresa. Apoyo Administrativo ferretería. Correo: zeddy97@yahoo.es

[3] Licenciado en Matemáticas, Docente de la URACCAN. Correo: osmartola@yahoo.es
} 


\section{Summary}

This research has applied the Critical Path Method (CPM) and the Evaluation Techniques and Program Review (PERT) in the administration of the project: "Development of technical and human capacities for the implementation of the regional development plan with identity in the Mines", executed in Siuna, during the year 2012, by the URACCAN. In the administration of the project study, we used a retrospective and quantitative approach, framed in the administrative techniques of the CPM and PERT project through WinQSB program.

According to the results, the entire project can be completed in 51.3 months, equivalent to 4 years and 3 months, with 12 critical activities, which means that a delay in any of these activities can cause a delay in the entire project. The total cost of the activities of this project is $617,863.00$ euros, and the total cost of the critical path is 231,585.00 euros. The project incurs into other costs, such as labor, transportation, consultation services, evaluation, among others, that are also part of the total project budget of 1,040,251.00 euros.

The project took longer to complete each of the activities, since in the initial planning it was scheduled to be finished in 3 years, which was not fulfilled because they had to request an extension of three months for full conclusion. The advantage in implementing the Critical Path Method is that it gave the perspective to have the project information organized and ready for any review; one of the main disadvantages is that all activities had to be organized regarding their immediate predecessors and to have knowledge of the method as well as the WinQSB program in order to avoid adverse outcomes.

Keywords: Administrative techniques; WINQSB Program; critical activities; critical path; immediate predecessors.

\section{Introducción}

La administración de proyectos implica una gran importancia, por lo que es útil en una diversidad de campos; desde proyectos espaciales, bancarios, sistemas computarizados, hidrocarburos, industria petroquímica, telecomunicaciones, en defensa nacional, entre otros. Los cambios tecnológicos, la necesidad de introducir nuevos productos al mercado, las cambiantes exigencias de los consumidores de productos, entre otras cosas, incrementan el fluido de operaciones en una organización, provocando que los métodos administrativos convencionales sean inadecuados, por esta razón la administración de proyectos es importante, ya que ofrece nuevas alternativas de organización y sirve para aprovechar de mejor manera los recursos críticos cuando están limitados en cantidad o tiempo de disponibilidad. 


\section{CIENCIAS SOCIALES}

Las micro empresas y negocios del municipio de Siuna enfrentan la dificultad de encontrar una técnica administrativa que ayude a comprobar y controlar un proyecto que involucra numerosas tareas interrelacionadas. La supervisión de estos proyectos requiere muchas habilidades administrativas, una de la cuales es mantener el proyecto acorde a un programa. Este estudio se realizó en la Universidad de las Regiones Autónomas de la Costa Caribe Nicaragüense URACCAN-Siuna con el objeto de aplicar el método de la ruta crítica (CPM) y las Técnicas de Evaluación y Revisión de Programas (PERT) en la administración del proyecto: "Desarrollo de capacidades técnicas y humanas para la implementación del plan de desarrollo regional con identidad en el sector de Las Minas".

El producto de la investigación será de mucha utilidad a la Universidad, porque a través de éste se mostrarán las técnicas administrativas de control para la administración de proyectos, que les facilite tomar decisiones en la ejecución de sus proyectos. Servirá a la sociedad como base de información a personas que estén interesadas en trabajar la temática, del mismo modo a la comunidad universitaria de nuestra Alma Mater, quedando un documento en la biblioteca del Recinto Universitario URACCANSiuna.

\section{Revisión de literatura}

\section{Proyecto}

Se define un proyecto como un conjunto de actividades interrelacionadas, en la que cada actividad consume tiempo y recursos (Taha, 2004, p.266). Un proyecto no es ni más ni menos que la búsqueda de una solución inteligente al planteamiento de un problema tendiente a resolver, entre tantas, una necesidad humana. Cualquiera sea la idea que se pretende implementar en la inversión, la metodología o la tecnología por aplicar, ella conlleva necesariamente a la búsqueda de proposiciones coherentes destinadas a resolver las necesidades de la persona humana (Sapag, 2003, p.4).

\section{Administración de proyectos}

Administrar un proyecto implica planificar, dirigir y controlar los recursos (personas, equipos y materiales) para cumplir con las restricciones técnicas, de costos y de tiempo para el proyecto (Diez, S/sP. 3).

Dicho en forma breve, el proceso de administración del proyecto significa planear el trabajo y después trabajar el plan para lograr el objetivo del proyecto. El esfuerzo principal en la administración de un proyecto tiene que estar centrada en establecer un plan de línea base que proporcione un plan de ruta para indicar cómo se logrará el alcance del proyecto a tiempo y dentro del presupuesto (Gido y Clements, Ibíd. P.11). 
En toda actividad es importante que se lleve a cabo un orden, un plan y administración de lo que se desea llevar a cabo, con esto nos damos cuenta de lo que podemos alcanzar, si nos hace falta algo o si vamos por el camino correcto, por otro lado, tomamos en cuenta todos los factores que pueden influir en el procedimiento que llevaremos para lograr nuestro objetivo, administrar nos acerca al éxito (Cabrera, 2009, P.4).

\section{Método de la Ruta Crítica CPM)}

El Método de la Ruta Crítica (CPM equivalente a la sigla en inglés Critical Path Method) es frecuentemente utilizado en el desarrollo y control de proyectos. El objetivo principal es determinar la duración de un proyecto, entendiendo éste como una secuencia de actividades relacionadas entre sí, donde cada una de las actividades tiene una duración estimada (Tobar, S/f, P. 1).

El Método de la Ruta Crítica es aplicable y útil en cualquier situación en la que se tenga que llevar a cabo una serie de actividades relacionadas entre sí para alcanzar un objetivo determinado (Andrade, 2012, p. 1).

El Método de la Ruta Crítica (Critical Path Method), es utilizado para administrar proyectos en que los tiempos requeridos para terminar las tareas individuales se conocen con certeza (Mathur y Solow, 1996, P. 493). Los proyectos terminados consisten en diversas tareas individuales, para comprobar los proyectos, primero debe identificar esas tareas (Ibid., p. 494).

Una red consiste en una colección finita de nodos y arcos. Un arco es una flecha que conecta un nodo de otro. En la administración de proyectos los nodos y arcos de la red de proyectos tienen un significado especial en el contexto del programa específico, dependiendo de qué tipo de enfoques estándar se utilice. (Ibíd., P. 497).

Una actividad crítica significa que un retraso en cualquiera de esas actividades ocasiona un retraso en todo el proyecto. Los retrasos del proyecto pueden producir costos adicionales, ingresos perdidos y/o incumplimiento de las obligaciones contractuales (Ibíd., p. 512).

\section{Técnicas de Evaluación y Revisión de Programas (PERT)}

El método PERT (Program Evaluation and Review Technique) es una metodología que a diferencia del CPM permite manejar la incertidumbre en el tiempo de término de las actividades (Tobar, Ibíd., P.4). 


\section{Ventajas y desventajas de las técnicas administrativas}

En la gestión de tiempos del proyecto, el método del camino crítico o CPM se utiliza a fin de detectar las tareas, sea que atrasen o adelanten la finalización del proyecto (Muiño, 2008, P. 1).

Si queremos entonces mejorar los plazos de un proyecto, debemos mejorar primero los plazos de las tareas del camino crítico, y a que mejorar plazos de tareas no críticas no adelanta la finalización. Con igual criterio al realizar el seguimiento de tiempos, será condición necesaria cumplir con los plazos de las tareas críticas para cumplir con el plazo total (Ibíd.).

Según López W. (2007), se ha demostrado que las técnicas de PERT, CPM y otras utilizadas en la Gerencia de Proyectos son claves para las funciones de planificación y control de proyectos. Estas permiten al gerente de proyecto entender la situación actual de cada actividad y saber cuáles son o no son críticas, además añade que el éxito de las empresas dependerá en gran medida de qué tan definida se presente cada actividad de un proyecto. Esto servirá de guía para todo proceso y facilitará la toma de decisiones eficientes y efectivas que estén fundamentadas en información y datos confiables. Según Rivera (2005), estas técnicas administrativas enseñan una disciplina lógica para planificar y organizar un programa detallado de largo alcance.

\section{Materiales y métodos}

La investigación es de tipo cuantitativa, y en ella se utilizó técnicas de entrevistas directas y encuestas, así como revisión documental para la recopilación de la información sobre la administración y ejecución del proyecto: "Desarrollo de capacidades técnicas y humanas para la implementación del plan de desarrollo regional con identidad en el sector de las Minas". Para el procesamiento de los datos se hizo uso de los siguientes programas: Microsoft Office Word 2013, WinQSB 2.o (Quantitative System Bussines) e IBM SPSS Statistics 21 (Statistical Package for Social Sciences) con el fin de garantizar el análisis estadístico de datos.

\section{Resultados discusión}

\section{Método de la ruta crítica al proyecto mediante el programa WinQSB}

\section{Paso No. 1: Identificar la lista de actividades del proyecto}

$\mathrm{Al}$ realizar la revisión documental del proyecto tuvimos como resultado una lista de actividades que identificamos mediante una letra mayúscula del alfabeto, según el orden en que estaban en el plan, las cuales las podemos visualizar en el siguiente cuadro: 
Cuadro No. 1. Actividades del proyecto

\begin{tabular}{|c|c|}
\hline Etiqueta & Descripción \\
\hline A & $\begin{array}{l}\text { Planificar y ejecutar una maestría en planificación y desarrollo regional sos- } \\
\text { tenible. }\end{array}$ \\
\hline B & $\begin{array}{l}\text { Desarrollar postgrado de especialización orientado a la transformación } \\
\text { económica, equitativa, sostenible y armónica entre los seres humanos y la } \\
\text { naturaleza. }\end{array}$ \\
\hline $\mathrm{C}$ & $\begin{array}{l}\text { Apoyar la realización de } 41 \text { investigaciones: } 5 \text { tesis de maestría, } 27 \text { monogra- } \\
\text { fías, } 3 \text { Multidisciplinaria y } 6 \text { Academia. }\end{array}$ \\
\hline D & Garantizar el ingreso de 260 nuevos estudiantes de pregrado becados. \\
\hline $\mathrm{E}$ & Organizar y asesorar las prácticas de campo de estudiantes de pregrado. \\
\hline $\mathbf{F}$ & $\begin{array}{l}\text { Establecer una plataforma virtual para articular encuentros presenciales y } \\
\text { virtuales. }\end{array}$ \\
\hline $\mathbf{G}$ & $\begin{array}{l}\text { Desarrollar un plan de divulgación para captar estudiantes de las comunida- } \\
\text { des. }\end{array}$ \\
\hline H & Curso de educación continua para la docencia. \\
\hline I & Garantizar acervo bibliográfico. \\
\hline$J$ & $\begin{array}{l}\text { Planificar y ejecutar un diplomado sobre desarrollo económico con identi- } \\
\text { dad. }\end{array}$ \\
\hline K & $\begin{array}{l}\text { Asesorar la elaboración y ejecución de planes de desarrollo de las } 16 \text { comu- } \\
\text { nidades. }\end{array}$ \\
\hline $\mathbf{L}$ & $\begin{array}{l}\text { Organizar intercambios de experiencias a nivel regional, nacional e interna- } \\
\text { cional. }\end{array}$ \\
\hline M & $\begin{array}{l}\text { Ejecutar un plan de capacitación para las } 16 \text { comunidades sobre redes, ma- } \\
\text { nejo productivo, planes de negocio, género, VIH/Sida. }\end{array}$ \\
\hline $\mathbf{N}$ & $\begin{array}{l}\text { Implementar una estrategia de trabajo con las mujeres de las } 16 \text { comunida- } \\
\text { des para el fortalecimiento del liderazgo femenino. }\end{array}$ \\
\hline 0 & $\begin{array}{l}\text { Acompañar al liderazgo comunitario en la realización de gestiones ante las } \\
\text { autoridades locales y otros actores sociales de la región. }\end{array}$ \\
\hline $\mathbf{P}$ & $\begin{array}{l}\text { Integrar al relevo generacional de los productores a fin de ir preparando la } \\
\text { cantera de campesinos y campesinas para asumir el liderazgo comunitario. }\end{array}$ \\
\hline Q & Definir estándares de calidad productiva. \\
\hline $\mathbf{R}$ & Definir una estrategia o mecanismo para la comercialización. \\
\hline S & Establecer a 120 familias con sistemas productivos sostenibles. \\
\hline $\mathbf{T}$ & Apoyar la organización de 5 redes comunitarias de comercialización. \\
\hline U & Apoyar la implementación de mecanismos de comercialización. \\
\hline V & Buscar e identificar variedades de plantas productivas criollas y nativas. \\
\hline
\end{tabular}




\section{CIENCIAS SOCIALES}

El orden lógico de las actividades visualizadas en el cuadro, ya estaba en la planificación inicial del proyecto, por lo tanto solamente se procedió a hacer la revisión documental para la realización del primer paso de nuestro estudio que consiste principalmente en identificar cada una de las actividades que conforman el proyecto en estudio.

\section{Paso No. 2: Estimación del tiempo para cada tarea}

De la entrevista realizada a los ejecutores del proyecto, sobre la planificación de las actividades asignadas y mediante la revisión documental, podemos apreciar en el cuadro 2 mostrado abajo, la estimación del tiempo real para cada tarea:

Cuadro No. 2. Tiempo asignado para cada actividad

\begin{tabular}{|c|c|}
\hline Etiqueta & Estimación de tiempo (meses) \\
\hline A & 5.8 \\
\hline B & 1 \\
\hline C & 4 \\
\hline D & 1 \\
\hline E & 1 \\
\hline F & 2.4 \\
\hline G & 2.8 \\
\hline H & 2 \\
\hline I & 1.5 \\
\hline J & 2.4 \\
\hline K & $\mathbf{1}$ \\
\hline L & 2 \\
\hline M & 3,5 \\
\hline N & 1 \\
\hline $\mathbf{~}$ & 4,6 \\
\hline P & 8 \\
\hline Q & 3,5 \\
\hline R & 6 \\
\hline S & 6 \\
\hline T & 6 \\
\hline U & 6 \\
\hline V & \\
\hline & \\
\hline
\end{tabular}


El tiempo asignado para cada una de las actividades del cuadro anterior fue el tiempo real de ejecución de las actividades del proyecto, puesto que el proyecto solamente cuenta con una macro planificación en la que incluye sólo el tiempo en que cada actividad debe iniciar y el tiempo en que debe terminar.

El personal encargado de ejecutar y controlar las actividades del proyecto, cumple en un $80 \%$ con el inicio programado de cada actividad, de acuerdo a la encuesta realizada (Ver figura. No. 1).

\section{Paso No. 3: Creación de la tabla de precedencia para el proyecto}

Según información proporcionada por el coordinador de cooperación externa podemos visualizar mediante el siguiente cuadro la relación de las actividades.

Cuadro No. 3. Tabla de precedencia del proyecto

\begin{tabular}{|c|c|c|}
\hline Etiqueta & $\begin{array}{l}\text { Estimación de } \\
\text { tiempo (meses) }\end{array}$ & $\begin{array}{l}\text { Predecesores } \\
\text { Inmediatos }\end{array}$ \\
\hline A & 5.8 & Ninguna \\
\hline B & 1 & Ninguna \\
\hline C & 4 & A \\
\hline D & 1 & Ninguna \\
\hline $\mathrm{E}$ & 4.5 & $C, D$ \\
\hline $\mathbf{F}$ & 1 & $A, B$ \\
\hline G & 2.4 & $A, B, D, E$ \\
\hline $\mathrm{H}$ & 2.8 & $\mathrm{D}$ \\
\hline 1 & 2 & $\mathrm{D}, \mathrm{H}$ \\
\hline J & 1.5 & Ninguna \\
\hline $\mathrm{K}$ & 2.4 & $\mathrm{~B}, \mathrm{D}, \mathrm{E}$ \\
\hline $\mathbf{L}$ & 1 & $B, E, J$ \\
\hline$M$ & 2 & $J, K, L$ \\
\hline $\mathrm{N}$ & 3,5 & $J, K, L, M$ \\
\hline 0 & 1 & $J, K, L, M, N$ \\
\hline $\mathbf{P}$ & 4,6 & $\mathrm{~K}, \mathrm{~L}, \mathrm{M}, \mathrm{N}, \mathrm{O}$ \\
\hline$Q$ & 8 & $\mathrm{~K}, \mathrm{~L}, \mathrm{M}, \mathrm{N}, \mathrm{O}, \mathrm{P}$ \\
\hline $\mathbf{R}$ & 3,5 & $\mathrm{~A}, \mathrm{~B}, \mathrm{C}, \mathrm{D}, \mathrm{E}, \mathrm{F}, \mathrm{G}, \mathrm{J}, \mathrm{K}, \mathrm{L}, \mathrm{M}, \mathrm{N}, \mathrm{O}, \mathrm{P}, \mathrm{Q}$ \\
\hline $\mathrm{S}$ & 6 & $\mathrm{~K}, \mathrm{~B}, \mathrm{~J}, \mathrm{Q}, \mathrm{R}$ \\
\hline$T$ & 6 & $Q, R$ \\
\hline u & 6 & $\mathrm{~K}, \mathrm{M}, \mathrm{N}, \mathrm{O}, \mathrm{P}, \mathrm{Q}, \mathrm{R}, \mathrm{S}, \mathrm{T}$ \\
\hline $\mathrm{v}$ & 6 & $C$ \\
\hline
\end{tabular}




\section{CIENCIAS SOCIALES}

El cuadro muestra cada una de las actividades con su tiempo y sus predecesores inmediatos, que son los que se utilizan para saber de qué manera se relacionan unas actividades con otras, es decir que actividad debe ejecutarse primero y cual después. Por ejemplo, la actividad A y B pueden iniciar sin ningún problema mientras que para iniciar la actividad $C$ se tuvo que haber ejecutado la actividad $A$.

\section{Paso No. 4: Cálculo del tiempo en la terminación del proyecto}

Con toda la información recopilada, es de nuestro conocimiento que el proyecto consta de 22 actividades, para lo cual utilizamos el programa WinQSB para la implementación del Método de la Ruta Crítica. A continuación se muestran por fases los datos obtenidos después de la implementación del Método de la Ruta Crítica en el programa $W$ inQSB, también se hace una descripción de cada fase.

\section{Fase No. 1. Introduciendo datos a WinQSB}

El cuadro que se muestra a continuación se representan los datos proporcionados por el coordinador de cooperación externa y los ejecutores de proyecto de la URACCAN, los cuales fueron visualizados en los cuadros anteriores:

Cuadro No. 4. Tabla completa del proyecto

\begin{tabular}{|c|c|c|c|c|}
\hline $\begin{array}{l}\text { Número de } \\
\text { actividad }\end{array}$ & $\begin{array}{c}\text { Etiqueta de } \\
\text { actividad }\end{array}$ & $\begin{array}{l}\text { Predecesores } \\
\text { Inmediatos }\end{array}$ & $\begin{array}{l}\text { Tiempo } \\
\text { norma }\end{array}$ & $\begin{array}{c}\text { Costo } \\
\text { normal }\end{array}$ \\
\hline 1 & $A$ & Ninguna & 5.8 & $€ 97.091,0$ \\
\hline 2 & B & Ninguna & 1 & $€ 36.569,0$ \\
\hline 3 & C & A & 4 & $€ 30.787,0$ \\
\hline 4 & D & Ninguna & 1 & $€ 275.166$ \\
\hline 5 & E & $C, D$ & 4.5 & $€ 4.875,00$ \\
\hline 6 & $\mathrm{~F}$ & $A, B$ & 1 & $€ 16.666,0$ \\
\hline 7 & G & $\mathrm{A}, \mathrm{B}, \mathrm{D}, \mathrm{E}$ & 2.4 & $€ 1.625,00$ \\
\hline 8 & $\mathrm{H}$ & D & 2.8 & $€ 14.515,0$ \\
\hline 9 & I & $\mathrm{D}, \mathrm{H}$ & 2 & $€ 5.417,00$ \\
\hline 10 & J & Ninguna & 1.5 & $€ 19.237,0$ \\
\hline 11 & K & $\mathrm{B}, \mathrm{D}, \mathrm{E}$ & 2.4 & $€ 7.248,00$ \\
\hline 12 & $\mathrm{~L}$ & $B, E, J$ & 1 & $€ 12.083,0$ \\
\hline 13 & M & $\mathrm{J}, \mathrm{K}, \mathrm{L}$ & 2 & $€ 6.250,00$ \\
\hline 14 & $\mathrm{~N}$ & $J, K, L, M$ & 3,5 & $€ 6.500,00$ \\
\hline 15 & 0 & $J, K, L, M, N$ & 1 & $€ 1.875,00$ \\
\hline 16 & $P$ & $\mathrm{~K}, \mathrm{~L}, \mathrm{M}, \mathrm{N}, \mathrm{O}$ & 4,6 & $€ 4.875,00$ \\
\hline
\end{tabular}




\begin{tabular}{|l|l|l|l|r|}
\hline $\begin{array}{c}\text { Número de } \\
\text { actividad }\end{array}$ & \multicolumn{1}{|c|}{$\begin{array}{c}\text { Etiqueta de } \\
\text { actividad }\end{array}$} & \multicolumn{1}{|c|}{$\begin{array}{c}\text { Predecesores } \\
\text { Inmediatos }\end{array}$} & \multicolumn{1}{|c|}{$\begin{array}{c}\text { Tiempo } \\
\text { norma }\end{array}$} & \multicolumn{1}{c|}{$\begin{array}{c}\text { Costo } \\
\text { normal }\end{array}$} \\
\hline 17 & $\mathrm{Q}$ & $\mathrm{K}, \mathrm{L}, \mathrm{M}, \mathrm{N}, \mathrm{O}, \mathrm{P}$ & 8 & $€ 2.500,00$ \\
\hline 18 & $\mathrm{R}$ & $\begin{array}{l}\mathrm{A}, \mathrm{B}, \mathrm{C}, \mathrm{D}, \mathrm{E}, \mathrm{F}, \mathrm{G}, \mathrm{J}, \mathrm{K}, \mathrm{L} \\
, \mathrm{M}, \mathrm{N}, \mathrm{O}, \mathrm{P}, \mathrm{Q}\end{array}$ & 3,5 & $€ 2.500,00$ \\
\hline 19 & $\mathrm{~S}$ & $\mathrm{~K}, \mathrm{~B}, \mathrm{~J}, \mathrm{Q}, \mathrm{R}$ & 6 & $€ 40.000,0$ \\
\hline 20 & $\mathrm{~T}$ & $\mathrm{Q}, \mathrm{R}$ & 6 & $€ 12.500,0$ \\
\hline 21 & $\mathrm{U}$ & $\mathrm{K}, \mathrm{M}, \mathrm{N}, \mathrm{O}, \mathrm{P}, \mathrm{Q}, \mathrm{R}, \mathrm{S}, \mathrm{T}$ & 6 & $€ 14.584,0$ \\
\hline 22 & $\mathrm{~V}$ & $\mathrm{C}$ & 6 & $€ 5.000,00$ \\
\hline
\end{tabular}

El cuadro anterior muestra de manera resumida toda la información que se requiere para la implementación del método de la ruta crítica al proyecto a través del programa $\mathrm{W}$ inQSB, donde se puede apreciar, el número y la etiqueta de las actividades correspondiente a la primera y segunda columna.

Los predecesores inmediatos mostrados en la tercera columna representan la relación de las actividades, es decir qué actividades preceden de otras. En el cuadro también se puede observar en las últimas columnas el tiempo y el costo normal para la ejecución de cada una de las actividades.

Con respecto al costo de las actividades se consideró necesario tomarlo en cuenta con el fin de determinar el presupuesto total de todas las actividades que conforman el proyecto en estudio, para luego hacer una comparación con el presupuesto programado en la planificación inicial del mismo. Se considera que es significativo plantear de una manera directa y resumida todas las actividades del proyecto con sus predecesores, tiempo y costo, con el fin de utilizar la información cuando sea necesario.

Se piensa que si en el proyecto se hubiera realizado este tipo de método para resumir la información del proyecto, se hubiese utilizado como una guía en la ejecución de las actividades con el fin de cumplir al máximo con lo planificado.

Fase No. 2. Análisis de las actividades del proyecto: "Desarrollo de capacidades humanas". Los resultados obtenidos de esta fase se presentan en el siguiente cuadro, donde podemos visualizar las actividades del proyecto con sus respectivos análisis. 


\section{CIENCIAS SOCIALES}

Cuadro No. 5. Análisis de las actividades del proyecto: "Desarrollo de capacidades humanas" mediante el CPM

\begin{tabular}{|c|c|c|c|c|c|c|c|c|}
\hline No. & Etiqueta & $\begin{array}{l}\text { Actividades } \\
\text { críticas }\end{array}$ & Tiempo & $\begin{array}{c}\text { Tiempo } \\
\text { de inicio } \\
\text { inmediato }\end{array}$ & $\begin{array}{c}\text { Tiempo } \\
\text { final } \\
\text { inmediato }\end{array}$ & $\begin{array}{c}\text { Ultimo } \\
\text { tiempo de } \\
\text { inicio }\end{array}$ & $\begin{array}{c}\text { Ultimo } \\
\text { tiempo de } \\
\text { terminación }\end{array}$ & $\begin{array}{c}\text { Tiempo de } \\
\text { retraso }\end{array}$ \\
\hline 1 & A & $\mathrm{Si}$ & 5,8 & 0 & 5,8 & 0 & 5,8 & 0 \\
\hline 2 & B & No & 1 & 0 & 1 & 13,3 & 14,3 & 13,3 \\
\hline 3 & C & $\mathrm{Si}$ & 4 & 5,8 & 9,8 & 5,8 & 9,8 & O \\
\hline 4 & D & No & 1 & 0 & 1 & 8,8 & 9,8 & 8,8 \\
\hline 5 & E & $\mathrm{Si}$ & 4,5 & 9,8 & 14,3 & 9,8 & 14,3 & 0 \\
\hline 6 & $\mathrm{~F}$ & No & 1 & 5,8 & 6,8 & 34,8 & 35,8 & 29 \\
\hline 7 & G & No & 2,4 & 14,3 & 16,7 & 33,4 & 35,8 & 19,1 \\
\hline 8 & $\mathrm{H}$ & No & 2,8 & 1 & 3,8 & 46,5 & 49,3 & 45,5 \\
\hline 9 & I & No & 2 & 3,8 & 5,8 & 49,3 & 51,3 & 45,5 \\
\hline 10 & J & No & 1,5 & 0 & 1,5 & 14,2 & 15,7 & 14,2 \\
\hline 11 & K & $\mathrm{Si}$ & 2,4 & 14,3 & 16,7 & 14,3 & 16,7 & 0 \\
\hline 12 & L & No & 1 & 14,3 & 15,3 & 15,7 & 16,7 & 1,4 \\
\hline 13 & M & $\mathrm{Si}$ & 2 & 16,7 & 18,7 & 16,7 & 18,7 & O \\
\hline 14 & $N$ & $\mathrm{Si}$ & 3,5 & 18,7 & 22,2 & 18,7 & 22,2 & O \\
\hline 15 & 0 & Si & 1 & 22,2 & 23,2 & 22,2 & 23,2 & 0 \\
\hline 16 & $P$ & $\mathrm{Si}$ & 4,6 & 23,2 & 27,8 & 23,2 & 27,8 & 0 \\
\hline 17 & Q & $\mathrm{Si}$ & 8 & 27,8 & 35,8 & 27,8 & 35,8 & 0 \\
\hline 18 & R & $\mathrm{Si}$ & 3,5 & 35,8 & 39,3 & 35,8 & 39,3 & 0 \\
\hline 19 & $S$ & Si & 6 & 39,3 & 45,3 & 39,3 & 45,3 & 0 \\
\hline 20 & $\mathrm{~T}$ & Si & 6 & 39,3 & 45,3 & 39,3 & 45,3 & 0 \\
\hline 21 & $U$ & Si & 6 & 45,3 & 51,3 & 45,3 & 51,3 & 0 \\
\hline 22 & V & No & 6 & 9,8 & 15,8 & 45,3 & 51,3 & 35,5 \\
\hline
\end{tabular}

El Cuadro anterior muestra el análisis de cada una de las actividades que conforma el proyecto indicado y las actividades críticas visualizadas en la tercera columna.

Las actividades que aparecen con la palabra "si" indica que es una actividad crítica y las que aparecen con la palabra "no" indican que no es crítica y por lo tanto tiene un límite de tiempo que se puede retrasar sin afectar el tiempo de finalización total del proyecto.

El reporte mostrado en el mismo cuadro, también contiene una columna para lo siguiente: 
- Columna 5 y 6: Los tiempos de inicio y terminación más inmediatos. Por ejemplo, para la actividad B el tiempo de inicio más inmediato es o meses y el tiempo de terminación más breve es 1 mes.

- Columna 7 y 8: Los últimos tiempos de inicio y terminación. Por ejemplo, para la actividad B es 13,3 meses y el último tiempo de terminación es 14,3 meses.

- Columna 9: El tiempo de retraso. Por ejemplo, la actividad B tiene 13,3 meses de retraso, indicando que la misma puede retrasarse hasta 13,3 meses, sin afectar la conclusión del proyecto.

Los tiempos de retraso visualizados en la columna 9 del cuadro No. 5 representan el límite de tiempo en que una actividad puede retrasarse sin afectar el tiempo total de ejecución del proyecto. Cada actividad, con un tiempo de retraso de o días es crítica, siendo las actividades $A, C, E, K, M, N, O, P, Q, R, S, T$ y U críticas, es decir que un retraso en cualquiera de estas actividades ocasiona retraso en la conclusión total del proyecto.

En el siguiente cuadro se puede observar los resultados del Método de la Ruta Crítica mediante el programa WinQSB.

Cuadro No. 6. Resumen del proyecto completo

\begin{tabular}{|c|}
\hline $\begin{array}{c}\text { Tiempo total de terminación del proyecto } \\
=51,3 \text { Meses }\end{array}$ \\
\hline Costo total del proyecto $=€ 617.863,00$ \\
Costo de la ruta crítica $=€ 231.585,00$ \\
Número de rutas críticas 170 \\
\hline
\end{tabular}

El informe indica que todo el proyecto puede finalizar en 51,3 meses con un costo total de las actividades de $€ 617.863,00$ y el costo total de la ruta crítica de $€ 231.585,00$.

Fase No. 3. Proyecto "Desarrollo de capacidades humanas" (Determinación del tiempo de las actividades).

En esta fase se muestra la red del proyecto que contiene los tiempos de inicio y terminación más inmediatos de cada actividad, los cuales aparecen en verde y los últimos tiempos de inicio y terminación pintados en morado, vea también que las actividades criticas aparecen marcadas en rojo en la red del proyecto y forman en conjunto una ruta crítica desde el principio hasta el final del proyecto. (Ver figura No. 2) 


\section{CIENCIAS SOCIALES}

Tiempo de finalización del proyecto $=51,3$ Meses

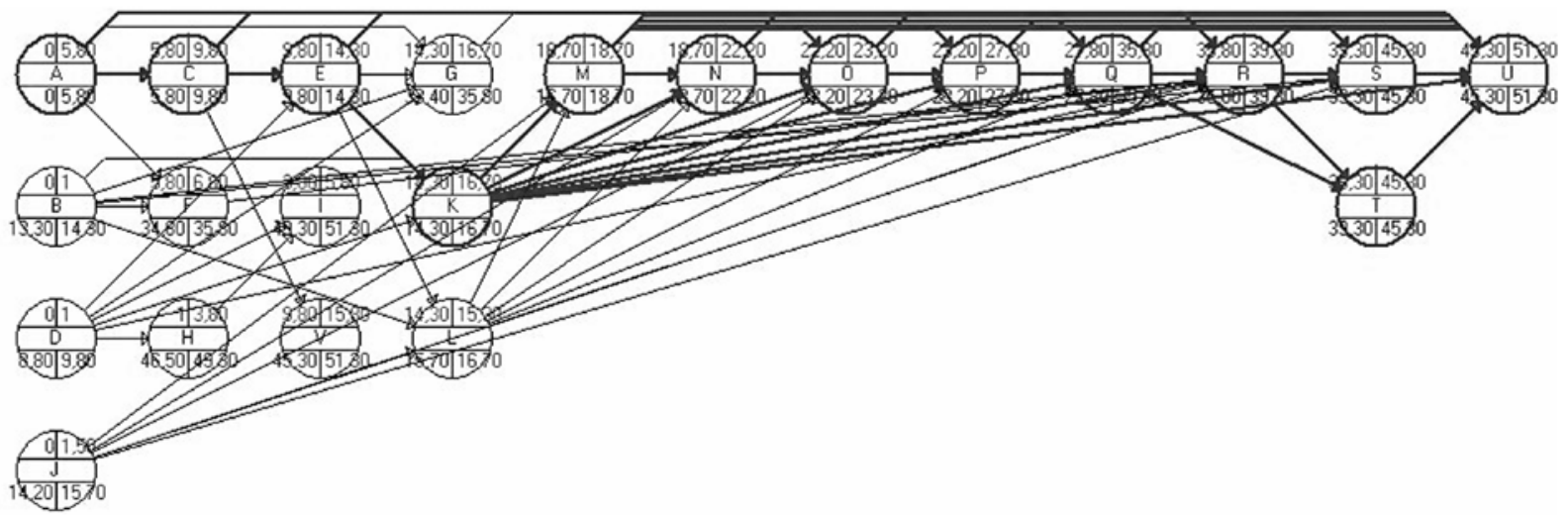

Figura No. 1. Representación gráfica del proyecto completo.

\section{Fase No. 4. Rutas críticas del proyecto: "Desarrollo de capacidades humanas"}

En general puede haber varias rutas críticas, como es el caso del proyecto en estudio que consta de 170 rutas, por lo tanto en esta fase se muestran a partir de la columna 2, solamente algunas de las rutas críticas que contiene este proyecto y las podemos apreciar en la red del proyecto.

Por ejemplo en la columna 2 se muestra la ruta crítica formada por las actividades $\mathrm{A}, \mathrm{C}, \mathrm{E}, \mathrm{K}, \mathrm{M}, \mathrm{N}, \mathrm{O}, \mathrm{P}, \mathrm{Q}, \mathrm{R}, \mathrm{S}$ y $\mathrm{U}$, indicando una secuencia de tareas críticas para el proyecto, que conecta el principio y el final del mismo.

Cuadro No. 7. Rutas críticas del proyecto "Desarrollo de capacidades humanas"

\begin{tabular}{|c|c|c|c|c|c|c|}
\hline 07-16-2013 & $\begin{array}{c}\text { Ruta } \\
\text { crítica } \\
\mathbf{1}\end{array}$ & $\begin{array}{c}\text { Ruta } \\
\text { crítica } \\
\mathbf{2}\end{array}$ & $\begin{array}{c}\text { Ruta } \\
\text { crítica } \\
\mathbf{3}\end{array}$ & $\begin{array}{c}\text { Ruta } \\
\text { crítica } \\
\mathbf{4}\end{array}$ & $\begin{array}{c}\text { Ruta } \\
\text { crítica } \\
\mathbf{5}\end{array}$ & $\begin{array}{c}\text { Ruta } \\
\text { crítica } \\
\mathbf{6}\end{array}$ \\
\hline 1 & A & A & A & A & A & A \\
\hline 2 & C & C & C & C & C & C \\
\hline 3 & E & E & E & E & E & E \\
\hline 4 & K & K & K & K & K & K \\
\hline 5 & M & M & M & M & M & M \\
\hline 6 & N & N & N & N & N & N \\
\hline 7 & O & O & O & O & O & O \\
\hline 8 & P & P & P & P & P & P \\
\hline 9 & Q & Q & Q & S & T & U \\
\hline
\end{tabular}




\begin{tabular}{|c|c|c|c|c|c|c|}
\hline $\mathbf{0 7 - 1 6 - 2 0 1 3}$ & $\begin{array}{c}\text { Ruta } \\
\text { crítica } \\
\mathbf{1}\end{array}$ & $\begin{array}{c}\text { Ruta } \\
\text { crítica } \\
\mathbf{2}\end{array}$ & $\begin{array}{c}\text { Ruta } \\
\text { crítica } \\
\mathbf{3}\end{array}$ & $\begin{array}{c}\text { Ruta } \\
\text { crítica } \\
\mathbf{4}\end{array}$ & $\begin{array}{c}\text { Ruta } \\
\text { crítica } \\
\mathbf{5}\end{array}$ & $\begin{array}{c}\text { Ruta } \\
\text { crítica } \\
\mathbf{6}\end{array}$ \\
\hline 10 & $\mathrm{R}$ & $\mathrm{R}$ & $\mathrm{R}$ & $\mathrm{R}$ & $\mathrm{R}$ & $\mathrm{R}$ \\
\hline 11 & $\mathrm{~S}$ & $\mathrm{~T}$ & $\mathrm{U}$ & $\mathrm{U}$ & $\mathrm{U}$ & \\
\hline 12 & $\mathrm{U}$ & $\mathrm{U}$ & & & & \\
\hline $\begin{array}{l}\text { Tiempo de } \\
\text { finalización } \\
\text { (meses) }\end{array}$ & 51,3 & 51,3 & 51,3 & 51,3 & 51,3 & 51,3 \\
\hline
\end{tabular}

\section{- Análisis de los resultados del proyecto}

El proyecto "Desarrollo de capacidades técnicas y humanas para la implementación del plan de desarrollo regional con identidad en el sector de las Minas", estaba planificado para terminar en 3 años; pero, por algunos problemas en la ejecución del mismo y otros factores relacionados con la naturaleza se tuvo que solicitar una prórroga de 3 meses, esto significa un retraso en todo el proyecto.

Al implementar el Método de la Ruta Crítica mediante el programa WinQSB al proyecto, se tuvo como resultado un tiempo de duración de 51,3 meses equivalente a 4 años y 3 meses, estos resultados nos reflejan que él mismo debió de planificarse para más tiempo y no para 3 años, por lo tanto se puede asegurar que si estas técnicas se hubiesen implementado al momento de la planificación inicial, los encargados de dar seguimiento al desarrollo del proyecto no hubieran tenido que recurrir a prórroga.

El costo total de las actividades del proyecto en estudio, mediante el CPM, haciendo uso del WinQSB, fue el mismo costo que se estimó en la programación inicial del proyecto el cual fue de $€ 617.863,00$. El tiempo de duración total del proyecto varía de acuerdo a la planificación inicial del mismo, mientras que el costo coincide exactamente con la planeación de determinado proyecto.

Se consultó específicamente el tiempo y el costo asignado para cada una de las actividades, así como la planificación y el presupuesto para realizar las respectivas gestiones al donante y a los ejecutores del proyecto, puesto que son principalmente los que se encargan de ejecutar las actividades que se les han asignado.

En cuanto al tiempo, podríamos decir que no se hizo una buena ejecución para cada una de las actividades de este proyecto, esto se puede justificar en el sentido de que finalizó el período de ejecución y se pidió una prórroga de tres meses para la conclusión total del mismo, producto de actividades tales como la maestría, mecanismos de comercialización y otras de fondo solidario que fueron las que más se retrasaron en el transcurso del desarrollo del proyecto. 
Con esto nos damos cuenta que el proyecto ejecutado por URACCAN necesitó más tiempo para poder concluir cada una de las actividades en tiempo y forma, es por esto que la administración de éste, a través del Método de la Ruta Crítica da como resultado un tiempo de finalización de 4 años y 3 meses.

Se considera que si se hubiera hecho una planificación utilizando el método de la ruta crítica a través del programa WinQSB se hubiera tenido todo el tiempo posible para terminar el proyecto en el tiempo estipulado en la programación inicial.

\section{Técnicas de Evaluación y Revisión de Programas (PERT)}

En el proyecto "Desarrollo de capacidades técnicas y humanas para la implementación del plan de desarrollo regional con identidad en el sector de las Minas", los tiempos de duración de todas las actividades se conocen con certeza, es por esto que aplicamos todos los pasos correspondientes al Método de la Ruta Crítica CPM.

En caso de que no se hubiese encontrado con exactitud los tiempos de ejecución de las actividades, hubiésemos aplicado los pasos del método PERT.

\section{Ventajas y desventajas de las técnicas administrativas CPM y PERT}

Después de haber implementado estas técnicas administrativas hemos encontrado las siguientes ventajas y desventajas, las cuales se describen a continuación:

\section{Ventajas}

Brindó la perspectiva para tener la información del proyecto de manera organizada y lista para cualquier revisión. Se utilizó el programa W inQSB para aplicar el Método de la Ruta Crítica con el objeto de minimizar el tiempo de implementación ya que aplicar este método de forma manual resulta muy tedioso.

Para aplicación del Método de la Ruta Crítica al proyecto no fue necesario tener muchos conocimientos matemáticos, pues se considera que el método es sencillo de implementar. Permitió dar un estimado de cuanto era el tiempo mínimo que debió haberse planificado el proyecto. Se pudo tener una percepción de las actividades que controlan la duración del proyecto (actividades críticas), ya que un retraso en cualquiera de estas afecta el tiempo de duración total del proyecto.

Se determinó el tiempo en que una actividad no crítica puede retrasarse sin afectar la conclusión total del proyecto. Este método permitió cuantificar los costos de ejecución por actividad y globales del proyecto, además mostró el costo que se puede perder si al implementar esta técnica no se cumple con el tiempo programado. 


\section{Desventajas}

Se tuvo que organizar todas las actividades con respecto a sus predecesores inmediatos. Fue necesario determinar el tiempo exacto de cada una de las actividades del proyecto, por lo tanto se hizo una recopilación de la información con cada uno de los ejecutores de proyecto y del coordinador de cooperación externa para hacer un consenso en general del tiempo, de acuerdo al tiempo que consumieron en la ejecución de las actividades.

Para aplicar estas técnicas administrativas fue necesario tener conocimiento de las mismas, así como del programa $\mathrm{W}$ inQSB, puesto que se deben seguir correctamente los pasos y una mala aplicación puede ocasionar que el programa no proporcione los datos exactos. El gráfico de redes del proyecto a través del programa W inQSB puede ser complicado y confuso de entender debido a la cantidad de actividades y las relaciones de interdependencia.

\section{Conclusiones}

- En la administración del proyecto "Desarrollo de capacidades humanas" a través del método de la ruta crítica (CPM) mediante el programa W inQSB se pudo constatar que el proyecto debió haberse planificado para 4 años y 3 meses y no para 3 años como estaba estipulado en la planificación inicial que se hizo en la URACCAN.

- El presupuesto asignado en la planificación de este proyecto, coincidió con el monto encontrado después de aplicar el método a través del software WinQSB.

- La ventaja de aplicar estas técnicas administrativas fue que los resultados del programa fueron óptimos y confiables para la administración del proyecto.

- La desventaja que se presentó al implementar el método de la ruta crítica fue que debíamos tener conocimiento tanto del método como del programa y aplicar correctamente los pasos de los mismos, ya que cualquier aplicación incorrecta nos hubiese provocado resultados adversos. 


\section{CIENCIAS SOCIALES}

\section{Lista de referencia}

Andrade Iván (2012). Ruta Crítica. (En línea). Disponible en http://www.Andradeivan. com/wp-content/uploads/2012/o4/EL-M\%C3Agtodo de la Ruta CriticaCr\%C3\%ADtica.pdf. (Consultado el 20 de enero 2013 a las 7:00 p.m.

Cabrera, Franco (2009). Importancia de la Administración de Proyectos (En línea). Disponible en: http://www.jcavilah.wordpress.com/2009/o3/o2/importanciade-la-administracion-de- proyectos/. (Consultado el 20 de enero 2013 a las 7:17 PM)

Diez, Pablo. (S/F). Operaciones 2 Proyectos y Redes. (En línea). Disponible en http://www. profesoere.ucv.cl/pablodiez/.t. (Consultado el 20 de enero 2013 a las 7:30 PM)

Gido J y Clements J. (1999). Administración Exitosa de Proyectos. Editorial International Thomson Editores, S. A, de C. V, México.

López, Walter (2007). Gerencia de Proyectos (En línea). Disponible en http://www1. uprh.edu/.../GERENCIA_DE_PROYECTOS_W alter_López. (Consultado el 10 de febrero 2013 a las 4:00 PM)

Mathur K y Solow D. (1996). Investigación de Operaciones. El arte de la toma de decisiones. Editorial: Prentice Hall Hispanoamericana, S. A, México.

Muiño, Adrián (2008). El método del Camino Crítico (CPM) tiene ventajas e inconvenientes. (En línea). Disponible en http://www.manuelgross.bligoo.com/content/ view/324407/El-metodo-del-

Camino- Crítico-CPM-tiene-vwntajas-e-inconvenientes.html. (Consultado el 20 de febrero 2013 a las 6:30 PM)

Rivera, Igor (2005). Investigación Operativa I. (en línea). Disponible en http://www. monografias.com.ingenieria. (Consultado. (Consultado el o2 de Marzo 2013 a las 4:02 PM)

Sapag, Nassir (2003). Preparación y Evaluación de Proyectos. 4ta edición. Editorial McGraw - Hill Interamericana, México.

Taha, Hamdy. (2004). Investigación de Operaciones. 7ma edición. Editorial Pearson Educación, S. A de C. V, México.

Tobar, Hugo (S/F). Investigación de Operaciones. (En línea). Disponible en: http: (Consultado el o3 de Abril 2013 a las 9:00 AM) 